\title{
Is the Second Twin at Risk in Vaginal Delivery?
}

\author{
Robert Derom \\ Association for Scientific Research in Multiple Births, Destelbergen, Belgium
}

In a normal delivery the second-born twin experiences a slight degree of hypoxia, which, as a rule, has no clinical importance. It is the oxygen debt everybody makes by running 30 metres.

From old epidemiologic data on comparative perinatal mortality it is well known that risk in vaginal delivery is higher in second than in first-born twins. One of the major reasons is hypoxia. However, no measurements of the frequency and the degree of the hypoxia have been made. Specific and accurate determination of hypoxia is the aim of this study.

Despite numerous studies on the respiratory function of the human placenta, relatively little is known about the adequacy of the oxygen supply to the fetus. Probably the best way to study this is to use an indirect approach: the investigation of anaerobic metabolism. A previous study was concerned with normal spontaneous deliveries (Derom, 1964). As a rule, no significant anaerobic metabolism could be demonstrated in the fetus at birth.

In the course of a systematic study of various obstetrical situations, it became clear that, in the case of twin deliveries, nature may have provided us with valuable material to investigate various degrees of intrauterine hypoxia. According to the fundamental work of Huckabee (1958), the measurements of both lactate and pyruvate in blood provide a reliable index of anaerobic metabolism. Only a fraction of lactate, called excess lactate by Huckabee (1958), correlates with the oxygen debt. This fraction $(\Delta \mathrm{XL})$ may be calculated, at any time $t$, according to the following equation:

$$
\mathrm{XL}=\left(\mathrm{L}_{1}-\mathrm{L}_{0}\right)-\left(\mathrm{P}_{1}-\mathrm{P}_{0}\right)\left(\mathrm{L}_{0} \mathrm{P}_{0}\right)
$$

where $\mathrm{L}_{1}$ and $\mathrm{L}_{0}$ refer to experimental and control lactate concentrations, and $\mathrm{P}_{1}$ and $\mathrm{P}_{0}$ to experimental and control pyruvate concentrations respectively. Any difference in excess lactate $(\Delta \mathrm{XL})$ of one twin as compared to the other may be calculated according to a modification of the above mentioned equation:

$$
\Delta \mathrm{XL}=\left(\mathrm{L}_{2}-\mathrm{L}_{1}\right)-\left(\mathrm{P}_{2}-\mathrm{P}_{1}\right)\left(\mathrm{L}_{1} / \mathrm{P}_{1}\right) .
$$

$\mathrm{L}_{1}$ and $\mathrm{P}_{1}$ refer to the concentrations of lactate and pyruvate in the first twin and $\mathrm{L}_{2}$ and $\mathrm{P}_{2}$ to the corresponding values in the second twin.

\section{Material and Methods}

The collection of blood and the determination of lactate and pyruvate have been described in a previous paper (Derom, 1964). The present series includes one case of cesarean section, performed under general anesthesia, and 13 sets of twins delivered vaginally without operative interference, except for case no. 8 in which vacuum extraction of the first baby was resorted to as a result of delay ( 2 hours) in the second stage. Neither analgesia nor anaesthesia was used with the exception of infiltration of the perineum. All breech deliveries were easy, the only manoeuvre employed was the manoeuvre of Bracht.

\section{Results}

Table 1 summarizes the most important clinical data concerning pregnancy and labor, and Table 2 the amount of excess lactate in umbilical vein blood.

Spontaneous twin deliveries may be considered as well-controlled experiments of nature; accordingly, when comparing the twins within each pair, only the differences between them should be taken into account for the statistical analysis of the results.

As suggested by the author and by others, the high lactate and pyruvate levels in umbilical blood in single births mainly, or even completely, result from diffusion out of maternal blood in which both these substrates are elevated because of the muscular activity incident of labor. If such diffusion of lactate and pyruvate from the mother to the fetus is approximately equal in both twins, any difference between twins is probably brought about by fetal metabolic processes. One may then reasonably assume that the increase in excess lactate in the second twin is not of maternal but of fetal origin.

The suggestion that the maternal influence may be approximately equal in both twins is strengthened by clinical observation. The muscular activity of the mother is generally no greater at the delivery of the second as compared to that of the first twin; occasionally the impression is gained that there is less bearing down for the expulsion of the second child. 
Table 1

Clinical Data of Pregnancy and Labor

\begin{tabular}{|c|c|c|c|c|c|}
\hline \multirow[t]{2}{*}{ Case no. } & \multirow[t]{2}{*}{ Parity } & \multicolumn{2}{|c|}{ Presentation } & \multirow[t]{2}{*}{ Time interval between deliveries (minutes) } & \multirow[t]{2}{*}{ Remarks } \\
\hline & & 1 & 2 & & \\
\hline 1 & 6 & B & V & 30 & \\
\hline 2 & 4 & V & V & 42 & Elective surgical induction of labor \\
\hline 3 & 7 & V & V & 5 & \\
\hline 4 & 5 & V & B & 25 & Neonatal death of second twin \\
\hline 5 & 5 & V & V & 10 & \\
\hline 6 & 11 & V & V & 16 & Pre-eclampsia \\
\hline 7 & 1 & V & V & 13 & Vacuum extraction of first twin \\
\hline 8 & 2 & V & B & 10 & \\
\hline 9 & 4 & V & V & 5 & \\
\hline 10 & 3 & B & B & 10 & \\
\hline 11 & 3 & B & $\mathrm{B}$ & 15 & \\
\hline 12 & 5 & V & $\mathrm{B}$ & 25 & \\
\hline 13 & 3 & B & B & 13 & \\
\hline 14 & 2 & V & B & 2 & Contracted pelvis, cesarean section \\
\hline
\end{tabular}

Hence, one may postulate that there is, for both deliveries, a comparable anaerobic metabolic activity in the mother and a comparable influence of the mother upon each fetus.

That the same intrauterine environment means similar concentrations of blood lactate and pyruvate is well illustrated in case no. 14 , in which delivery was accomplished by cesarean section; the twins were born immediately one after the other. In both children the values of lactate and pyruvate are almost identical. This shows that, when birth takes place under exactly the same circumstances, no significant differences in the levels of lactate and pyruvate are found.

\section{Table 2}

Excess Lactate in Umbilical Vein Blood

\begin{tabular}{lc}
\hline Case no. & Excess lactate $(\mathrm{mg} / 100 \mathrm{ml})$ \\
\hline 1 & +27.3 \\
2 & +23.6 \\
3 & +0.4 \\
4 & +7.9 \\
5 & +11.0 \\
6 & +4.6 \\
7 & +0.9 \\
8 & +7.0 \\
9 & +13.6 \\
10 & +2.6 \\
11 & +21.0 \\
12 & +2.0 \\
13 & +4.7 \\
Mean, \pm standard error & $+9.74 \pm 2.5$ \\
$14^{*}$ & +0.4 \\
\hline
\end{tabular}

Note: ${ }^{*}$ Cesarean section
Thus, available evidence points toward a fetal or endogenous accumulation of excess lactate in the second twin. This, in turn, means a raised anaerobic metabolism or an inadequate supply of oxygen to the tissues. The fetal hypoxia seems to be limited to the second twin. Indeed, in the first twin, the relationships of lactate and pyruvate are entirely comparable to those found in normal singleton deliveries, in which, as a rule, no significant fetal production of excess lactate could be demonstrated.

Beside the order of delivery, the only factor that apparently determines the hypoxia is the period of time between deliveries. When both twins are born immediately one after the other, as in case no. 14, born 2 minutes apart, no difference is found between the partners. Significant quantities of excess lactate (i.e., $1 \mathrm{mEq}$. per liter or $9 \mathrm{mg}$. per $100 \mathrm{ml}$ ) may be present in the second twin after varying periods of time. The highest amounts are found when approximately 30 minutes have elapsed between the deliveries. However, there is no fixed relationship between the degree of hypoxia and the period of time separating the deliveries. The presentation of the second twin, seemingly, has no influence on the result. As a matter of fact, hypoxia may be present with cephalic or with breech presentation. One of the children died in the neonatal period. The case history reads as follows:

The mother was a 35-year-old gravida v, para iv. She underwent regular prenatal care, but the presence of twins was not recognized. Labor started spontaneously at 32 weeks' gestation. Clinically, labor and delivery were normal. The second twin, a male who weighed 2.170 grams, was born in breech presentation 25 minutes after the first twin. Bracht's maneuver was used. The child cried immediately. His record does not mention any abnormalities during the first 3 days. In particular, no symptoms of meconium ileus were noted. The 4 th day the child had 
repeated attacks of cyanosis. No definite diagnosis could be established at that time. His condition deteriorated gradually thereafter and he died on the 6th day. Pertinent findings at necroscopy were large quantities of amniotic debris in one of the five pulmonary lobes and definite signs of fibrocystic disease of the pancreas.

Intrauterine hypoxia, as suggested by the aspiration of large quantities of amniotic fluid, may have been a contributory of even the chief cause of death in this neonate. Although the increase in excess lactate in Twin B as compared to Twin A was, in this case, rather low, it should be noted that the lactate/pyruvate ratio was abnormally high in the first twin (normal range 5 to 19.5). This suggests moderate to severe hypoxia. The hypoxia must have been even more pronounced in the second twin and may have reached a degree severe enough to influence his chances of survival.

\section{Comment}

After the birth of the first twin there must be diminution in the size of the placental site resulting in an impairment of the placental circulation. It should be remembered that the reduction of the placental site is sometimes followed by partial separation of the placenta. The importance of this phenomenon as a possible cause of hypoxia in the second twin has been pointed out by several authors, among them Eastman and MacLane (1931), Morison (1963), and Potter (1952). This mechanism is in accordance with our finding that, but for one probable exception, hypoxia cannot be demonstrated in the first twin, whereas it is frequently present in the second one.

There seems to be a higher risk of hypoxia in the second twin. MacDonald has made a special study of birth asphyxia in a series of 140 strictly comparable cases in which both babies were delivered spontaneously by the vertex. In double spontaneous vertex deliveries the second twin is, on the average, slower to breathe than the first. Thus, clinical data are in accordance with our biochemical findings.

\section{Conclusions}

Determinations of lactate and pyruvate in umbilical vein blood were performed in 14 cases of twin deliveries. Both babies were born spontaneously in 13 instances and by cesarean section in one instance. In double spontaneous deliveries the mean lactate/pyruvate ratio is the same as in normal single deliveries in the first twin, but it is significantly raised in the second. Assuming that, at the time both infants are born, the mother is performing approximately the same amount of muscular work and, consequently, approximately the same concentrations of blood lactate and pyruvate are present on the maternal side of the placenta, the elevated lactate/pyruvate ratio in the second twin suggests the occurrence of fetal hypoxia. If this assumption holds true, one may calculate the amount of endogenous excess lactate in the blood of the second twin, starting from the values of lactate and pyruvate in the first twin. Severe fetal hypoxia may be one of the reasons that perinatal mortality is comparatively raised in the second twin. The mean amount of hypoxia in the second twin has no clinical significance.

\section{$\overline{\text { Acknowledgment }}$}

Shortened version of an article published in the American Journal of Obstetrics and Gynecology (Derom, 1965).

\section{References}

Derom, R. (1965). Anaerobic metabolism in the human fetus. II. The twin delivery. American Journal of Obstetrics and Gynecology, 92, 555-561.

Derom, R. (1964). Anaerobic metabolism in the human fetus. I. The normal delivery. American Journal of Obstetrics and Gynecology, 89, 241-251.

Huckabee, W. E. (1958). Relationship of pyruvate and lactate during anaerobic metabolism. I. Effects of infusing of pyruvate or glucose and hyperventilation. Journal of Clinical Investigation, 37, 244-254.

Huckabee, W. E. (1958). Relationship of pyruvate and lactate during anaerobic metabolism. II. Exercise and formation of $\mathrm{O} 2$ debt. Journal of Clinical Investigation, 37, 255-263.

Eastman, N. J., \& MacLane, C. M. (1931). Foetal blood studies. II. The lactic acid content of umbilical cord blood under various conditions. Bulletin of the Johns Hopkins Hospital, 48, 261.

Potter, E. L. (1952). Pathology of the fetus and the newborn (p. 180). Chicago, IL: Year Book Publishers.

MacDonald, R. R. (1962). Management of the second twin. British Medical Journal, 1, 518-522.

Morison, J. E. (1963). Foetal and neonatal pathology (p. 160). London: Butterworth \& Company. 